\title{
DISTRIBUCIÓN DE LOS ESCENARIOS DEPORTIVOS EN LA CIUDAD DE MEDELLÍN: CONDICIONES DE POSIBILIDAD PARA SU ACCESO PARA LA MEJORA DE LA CUALIDAD DE VIDA DE LOS CIUDADANOS
}

\author{
DISTRIBUIÇÃO DE INSTALAÇÕES ESPORTIVAS NA CIDADE DE MEDELLÍN: \\ CONDIÇÕES DE POSSIBILIDADE DE CONTRIBUIR PARA A MELHORIA DA \\ QUALIDADE DE VIDA DOS CIDADÃOS.
}

DISTRIBUTION OF SPORTS SCENARIOS IN THE CITY OF MEDELLÍN: CONDITIONS OF POSSIBILITY FOR ACCESSING THEM IN ORDER TO IMPROVE CITIZENS' LIFE QUALITY.

José Fernando Tabares Fernandez*

Palabras clave: Derechos humanos. Equipo deportivo. Políticas públicas. Indicadores sociales.

Resumen: Se presentan los resultados de la aplicación del Índice de desarrollo y acceso deportivo (IDAD), realizado como componente de la investigación Los escenarios deportivos y las Unidades de Vida Articulada (UVA) en la dinámica activa de la ciudad, que buscó establecer la relación entre estos y objetivos sociales. Se trabajó con una perspectiva cuanti-cualitativa, con el IDAD, conversaciones, talleres, formatos y socializaciones. El texto se ocupa del IDAD. La investigación logró evidenciar la importancia de la relación entre acceso y distribución para la equidad, como uno los principales asuntos problemáticos en relación con objetivos de tipo social y contribuyó con propuestas y posibles rutas para el fortalecimiento de la política pública del sector en la ciudad, desde los escenarios deportivos como condición de posibilidad.

Palavras chave: Direitos humanos. Equipamentos esportivos. Políticas públicas. Indicadores sociais.
Keywords: Human rights. Sports equipment. Public policies. Social indicators.
Resumo: Este artigo apresenta os resultados da implementação do Índice de Desenvolvimento e Acesso Desportivo (IDAD), realizados como um componente da investigação "Os recintos esportivos e Unidades de Vida Articulada" (UVA) ${ }^{1}$ na dinâmica ativa da cidade, que procura estabelecer a relação entre esses e os fins sociais. Trabalhamos com uma perspectiva quanti-qualitativa com o IDAD, palestras, workshops, formatos e socializações. Este artigo trata do IDAD. Nesse contexto, a pesquisa conseguiu evidenciar a importância da relação para acesso e distribuição como um dos principais problemas relacionados aos objetivos sociais e contribuiu com propostas e possíveis caminhos para fortalecer as políticas públicas do setor na cidade, a partir dos espaços desportivos como condição de possibilidade.
Abstract: The article presents the results of the application of the Development Index and Sports Access (IDAD) under the study Sports Scenarios and the Articulated Life Unit (UVA) in the city's active dynamics, which sought to establish the relationship between these and social objectives. We worked under a quantitative-qualitative perspective, using the IDAD, conversations, workshops, formats, and socializations. The present text addresses the IDAD. In this context, the research was able to highlight the importance of the relationship between access and distribution for equity as one of the main problems related to social objectives and contributed with proposals and possible routes for strengthening the sector's public policy in the city based on sports scenarios as a condition of possibility.
*Universidad de Antioquia. Medellín, Colombia.

E-mail: josef.tabares@udea.edu.co

Recebido em: 13-04-2016 Aprovado em: 11-04-2017

(c) (1) (8) Licence 


\section{INTRODUCCIÓN}

La ciudad de Medellín, es fundada en año 1616 como resguardo de San Lorenzo de Aburra. Se caracterizó a lo largo del Siglo XX como centro fabril, gracias a los rendimientos de la minería del oro que se desarrolló desde principios de Siglo XIX, actividad que fue seguida por la siembra y recolección del café con características industriales.

En la actualidad, Medellín crece y se consolida como una ciudad de servicios, de turismo, de moda, estética e innovación. Desplaza de esta forma su vocación textil y manufacturera, su preponderante economía industrial y atiende así a los procesos de liberalización de los mercados mundiales, articulándose a la economía de los servicios transnacionales.

Medellín cuenta de acuerdo con cifras del 2015, con 2.464.322 habitantes, distribuidos desde lo político administrativo en 16 comunas y cinco corregimientos (zona rural). La ciudad, se comunica con los municipios cercanos mediante autopistas, túneles y líneas de Metro, que conjuntamente con líneas de Metrocable (telesférico), Metroplus y el tranvía, ofrecen un importante proyecto de transporte público para los habitantes de la ciudad.

En los procesos de transformación, destacan su apuesta turística y de servicios, la construcción y adecuación de parques, bibliotecas, museos y recuperación del espacio público y edificios emblemáticos, además de la construcción y adecuación de equipamientos deportivos, que como nuevos escenarios, consolidan la oferta de la ciudad para ser más competitiva en el contexto de globalización, que exige escenarios urbanos de digna presentación al mercado internacional.

En este contexto, el municipio de Medellín ha hecho un importante esfuerzo para edificar una importante infraestructura deportiva. Los Juegos Suramericanos en el 2010, marcaron un hito en la construcción y renovación de muchos de los escenarios deportivos de la ciudad. Pero además, en el marco de la política pública del deporte y la recreación desde el 2005, se apuesta por la dignificación de los escenarios deportivos existentes y la construcción de otros muchos, como contribución a la garantía del derecho al deporte

En este contexto, el deporte, en sus diferentes manifestaciones, es reconocido como derecho desde la Constitución Política de 1991, en su artículo 52 y reglamentado por la Ley 181 de 1995. Desde entonces se han implementado diferentes estrategias para su garantía, pero no se tienen estudios que permitan establecer su situación actual.

En 2009 se presentó el Plan decenal del deporte, la recreación y la actividad física para el desarrollo humano, la convivencia y la paz 2009-2019 como horizonte de posibilidad. Sin embargo, la pregunta por la relación que puede establecerse entre el campo y las líneas orientadoras del Plan, no parecen ser muy coherentes. En este contexto, no parece haber otro tipo de indicador que permita relacionar el deporte como derecho con otros asuntos de mayor complejidad y dado que la infraestructura deportiva es uno los indicadores por excelencia, debería tener una mayor atención.

En el transcurso de la última década, Medellín para la implementación de la Política Pública local para el deporte, la recreación y la actividad física, ha desarrollado un proyecto sostenido en el tiempo que ha permitido consolidar una importante propuesta. El ofrecimiento de diversas prácticas y dispositivos para la ciudadanía, las alianzas con otros actores de la administración municipal, el diseño de indicadores para establecer avances y logros, además 
de una iniciativa para la construcción, adecuación y mantenimiento de escenarios deportivos y recreativos para la ciudad, como posibilidad para la garantía del derecho al deporte y la recreación, entre otros.

La investigación, se inscribe en este último ítem. Buscó dar cuenta de rastros desde los cuales pudieran establecerse relaciones entre escenarios deportivos y beneficios que generan para la vida de los ciudadanos y ciudadanas.

El objetivo fue establecer la relación entre los equipamientos deportivos administrados por el Instituto de Deportes y Recreación de Medellín (INDER) ${ }^{1}$ y su contribución al bienestar de la ciudadanía. Es decir, el impacto social de la infraestructura con relación en temas tales como participación, emprendimiento y resiliencia. En este contexto, se buscó, como primera instancia, establecer la existencia de la infraestructura en los territorios y la posibilidad de acceso que tienen las personas, de acuerdo con el lugar donde habitan, como asunto fundamental para hablar de beneficios personales y sociales.

En este primer apartado, la investigación buscó criterios que permitieran complementar la cuantificación de los escenarios, con una mirada que los concibe como territorios con dinámicas propias favorecedoras u obstaculizadoras de procesos de inclusión y acceso. En este sentido, la propuesta metodológica se orientó tanto a dar cuenta del número y distribución, como de las dinámicas de gestión y funcionamiento de los mismos. Para ello, se usaron estrategias tanto cuantitativas como cualitativas. En relación con este asunto, se adelantó un trabajo correlacional que buscó establecer la relación entre el número de equipamientos deportivos y el número de habitantes en cada una de las comunas de la ciudad, mediante la aplicación del Índice de acceso y desarrollo deportivo (IDAD).

Sobre esta temática, pueden mencionarse trabajos tales como "Gestión implementación y seguimiento. Sistema de indicadores. El Sistema de Indicadores sociales INDER alcaldía de Medellín, 2009-2010" (INSTITUTO DE DEPORTE Y RECREACIÓN DE MEDELLÍN INDER, 2010). La práctica deportiva y su relación directa con el uso de los escenarios públicos y privados del barrio Castilla de la ciudad de Medellín (STUART RENDÓN, 2011), orientados fundamentalmente al número de usuarios, su caracterización y sus prácticas. En el INDER Medellín se destacan los trabajos: "Usos y prácticas de los escenarios deportivos administrados por el INDER Medellín" (INSTITUTO DE DEPORTE Y RECREACIÓN DE MEDELLÍN INDER, 2012), "Escenarios deportivos y recreativos INDERMedellín y Espacios públicos que transforman ciudad" (INSTITUTO DE DEPORTE Y RECREACIÓN DE MEDELÍN INDER, 2014), en los que se hicieron inventarios de equipamientos, su categorización y el análisis de su uso por parte de los diferentes actores sociales.

También se registran algunos trabajos sobre la temática, como el censo y distribución de equipamientos culturales (MOLINA, 2007); (MELO; PERES, 2009) y (TABARES; MOLINA, 2013), que buscaron visibilizar asuntos de equidad y acceso, de acuerdo con criterios socio espaciales y político administrativos.

En el desarrollo de la investigación, la pregunta por la incidencia (impacto) de la infraestructura deportiva en temas de emprendimiento, participación y resiliencia, requirió del análisis de aspectos desde los cuales hacer visible esta relación. En temas de impacto, es

1 De acuerdo con la Ley n. 181 de 1995, del deporte, se crea el Sistema Nacional del Deporte, que desde lo gubernamental contempla la instancia nacional representada por COLDEPORTES; la departamental (Los departamentos son la forma Político administrativa como se divide el país) cuya instancia son los INDEPORTES y en lo municipal, están los INDER, que son los encargos en lo local, es decir, los municipios. 
decir, de incidencia directa de un cierto asunto (en este caso escenarios deportivos) sobre procesos de tipo social, los rastros deben ser lo suficientemente amplios para ir más allá (que no sustituir) de los indicadores del tipo número de habitantes por metros cuadrados de escenarios. Si bien ellos pueden mostrar una parte de la posibilidad real de incidencia, se avanza hacia la inclusión de otros recortes de la realidad planteando indicadores más pertinentes. ¿Qué aspectos deberían tenerse en cuenta? ¿Qué tipo de indicador sería el más pertinente? ¿Qué parte de la realidad sería la más adecuada como recorte para evidenciar dicha incidencia? En últimas, la pregunta por los indicadores.

\section{LAS POLÍTICAS PÚBLICAS COMO OBJETO}

De acuerdo con Roth (2008), las políticas públicas representan en la actualidad un objeto de estudio, que al igual que los demás, encuentra explicación desde variadas perspectivas que buscan posicionar marcos referenciales para su estudio. Según el autor, es la perspectiva empírica la que ha predominado en este campo, aunque otras como el post empirismo, han problematizado la utilidad de las pruebas empíricas para este campo de estudios.

De acuerdo con Guba (1990), el planteamiento de los paradigmas de las ciencias sociales se trabaja desde tres referentes fundamentales: lo ontológico, lo epistemológico y lo metodológico (lo primero, referente a cómo se define la naturaleza de la realidad, lo segundo como la relación existente entre el saber y el investigador y finalmente la metodología como la manera como se descubre el conocimiento).

En esta dirección, el marco de análisis elegido representa una reducción de la realidad, una captura de una parte de ella, que no representa su complejidad. Es por ello que ninguna perspectiva, en ella misma, está en capacidad hacerlo.

En esta línea de análisis, la referencia de Prada $^{2}$ a Zemelman (1989), es ilustrativa para el tema tratado, cuando afirma que son: "[...] espacios cerrados por el indicador desde la visualización abierta por la huella de lo dado; es decir, que supone la problemática de las relaciones establecidas entre realidad y representación" (ZEMELMAN, 1989, p. 7).

Así entonces, dos son las opciones que aparecen para la construcción de indicadores. La tradicional, que lo plantea desde una prescripción teórica supuesta, el recorte de la realidad de acuerdo con una forma y la fijación de una realidad preestablecida de antemano. La otra, denominada epistemológica, se propone desde una apertura a los campos de posibilidades de la realidad, su articulación con los procesos reales considerados de acuerdo con la necesidad de concreción y la incidencia de las fuerzas sociales concurrentes como alternativas de la praxis.

De esta manera, la contradicción se presenta entre dos tipos de práctica: la analítica (mathesis) que corresponde a una práctica apropiadora-simbolizadora de la realidad, y la dialéctica, transformadora-simbolizadora de acuerdo con un procedimiento social articulador de los procesos existentes en un espacio concreto histórico cultural.

Desde la primera (analítica), de acuerdo con la ciencia experimental, se ha procedido de la siguiente manera: 
Aísla los objetos de estudio del contexto histórico-social donde se dan de manera objetiva, descompone sus objetos de estudio en unidades de análisis elementales desarticuladas entre sí, contrasta sus hipótesis empíricas individualizadas con las unidades de análisis convertidas en variables. Una vez que deductivamente ha encontrado un supuesto espacio investigable, ha intentado generalizar sus descubrimientos al espacio real. (ZEMELMAN, 1989, p.9).

Para esta perspectiva, los datos y los indicadores cumplen una función fundamental: "[...] son los hechos experimentales los que validan o invalidan una forma de conocimiento" (ZEMELMAN , 1989, p.9).

De acuerdo con este mismo autor, los diagnósticos tradicionales desconocen que las personas son los sujetos sociales, para nuestro caso, de los procesos que se dinamizan desde los escenarios deportivos para alcanzar objetivos sociales. O sea, más allá de usuarios que aparecen registrados en los reportes mensuales y anuales y de la relación que pueda establecerse entre equipamientos y número de habitantes, es importante comprender la condición de los sujetos sociales y su importante papel en la dinamización del sector. Es decir, en razón de este carácter, representa el elemento de articulación de la realidad a través de sus diferentes prácticas sociales. En referencia a cómo se aborde el análisis de la realidad, dos son las posibilidades que aparecen. Una, corresponde a la perspectiva que actúa por medio de un análisis lineal y estático de las distintas esferas de la realidad, aislando cada una de ellas. La otra corresponde a una apuesta por el estudio de la relación entre las esferas de la realidad. La propuesta sería la inclusión de las esferas política, cultural y psicosocial, en los diagnósticos, buscando explicitar las potencialidades de las alternativas contenidas en una situación concreta (ZEMELMAN, 1989). La posibilidad de los indicadores de impacto como reconstrucción de un campo de posibilidades.

En el campo del deporte, sus prácticas, a pesar de ser reconocidas como derecho, no encuentran rastros que permitan establecer su situación real y cuáles los factores que permitan su plena garantía (OLIVEIRA; TAFFAREL; BELÉM, 2014). Una realidad como la de Medellín, diversa y heterogénea, se enfrenta a la necesidad de identificar cuáles son los requerimientos actuales. Uno de ellos, imprescindible, los escenarios deportivos y la posibilidad de acceso para la ciudadanía. En este contexto, la propuesta de Mussino (1997), retomada por Oliveira, Taffarel e Belém (2014), del software y el hardware, referido este último a las instalaciones, da luces para avanzar.

De acuerdo con la propuesta de los autores, en referencia al hardware, se presentan propuestas socio-espaciales de censos de escenarios e información de la población (estrato, edad) que los circunda. Además de la georreferenciación, que ya existe en Medellín y de los metros cuadrados en escenarios en relación con el número de habitantes.

En este marco, la investigación sobre los escenarios deportivos y UVAS del INDER Medellín, se propone avanzar hacia la construcción de indicadores más desde la lectura del campo de posibilidades que de una cifra producto de un indicador planteado con anterioridad. Es decir, de acuerdo con la propuesta de Zemelman (1989), que parta de una concepción dinámica de la realidad. Desde aquí, el uso crítico de la teoría consiste en una problematización de los fenómenos y sus diversas conceptualizaciones, de modo tal que permita definir un campo de relaciones posibles, antes que reducirlas a los contenidos expresos en los conceptos y a las jerarquizaciones propuestas por los cuerpos teóricos. 
Desde esta postura, el contenido específico de cada uno de los niveles de lo real se alcanza solamente en el contexto que define la articulación específica de estos niveles en un recorte espacial y temporal específico, lo cual sustituye la formulación de hipótesis por un razonamiento reconstructivo en busca de la concreción (ZEMELMAN, 1989).

Cuando se intentan justificar beneficios sociales a partir de un equipamiento, en este caso deportivo, la pregunta que surge es ¿cuál es la dinámica en doble vía que posibilita que puedan establecerse relaciones propicias en tal sentido?

En esta dirección, la relación entre escenarios y habitantes, de acuerdo con su lugar residencia, puede ser un primer criterio de posibilidad. Es decir, el número de escenarios deportivos y número de habitantes que hay en la ciudad, como un primer criterio sobre la posibilidad de acceso. Pero además, qué relaciones pueden hacerse con otro tipo de indicadores cuyo objeto está más orientado al tema de las condiciones de vida. Es decir, la equidad en el acceso. Es en este contexto que se propone el IDAD. Con todas sus limitaciones, pero también como aporte para poner a dialogar la infraestructura deportiva con otros asuntos de ciudad, buscando evidenciar que su sola presencia no garantiza, en sí misma, una contribución a la vida de las personas.

\section{METODOLOGÍA}

Para este estudio, se adelantaron procesos de diseño de indicadores para los diferentes asuntos que conciernen al funcionamiento de un programa como el de escenarios deportivos del INDER Medellín. En esta dirección aspectos como la participación de la comunidad en la gestión fueron tratados. Para el caso del presente texto, se presentan los resultados concernientes al ejercicio inicial que correspondió a la identificación, clasificación y organización de los escenarios deportivos administrados por el INDER Medellín. Para ello se hizo un trabajo de identificación, registro, caracterización y ubicación de los mismos, a partir de la base de datos actualizada al momento de la investigación. En la búsqueda de dar cuenta no solo de la existencia, sino además de su ubicación en la ciudad y de la posibilidad de acceso de los habitantes desde el criterio de cada uno de los territorios, se definió que fuera con forma político-administrativa en se organiza la ciudad, es decir las Comunas y Corregimientos. Es decir, más allá de un número estático, los equipamientos son concebidos como rastros de condiciones de posibilidad, más centrados en las personas (actores sociales) que en su número o la asistencia a ellos. Asuntos como el acceso, la participación y en general la dinámica de los territorios (escenarios), deben ser vistas como realidad compleja con dinámicas vivas que se construyen en el día a día, condicionadas por la circunstancias propias de cada barrio, comuna y corregimiento.

Para efectos del presente texto, se enfatiza sobre la necesidad tanto de identificar el número de escenarios, como su ubicación en cada una de las comunas, relacionándolo además con el número de habitantes. En términos de acceso, aspecto fundamental para cualquier relación entre los equipamientos deportivos y sus posibles contribuciones a objetivos de bienestar humano, un primer criterio a ser tenido en cuenta es el número equipamientos y su relación con el número de habitantes como criterio. Para ello se hizo usó del método correlacional, tipo de investigación descriptiva, por representar una condición existente. Detalla de manera cuantitativa, el grado de relación de dos o más variables cuantificables. Es decir, el método correlacional permite indagar hasta qué punto las alteraciones de una variable dependen de las alteraciones de otra. (MOLINA et al. 2007). 
El estudio adaptó el IDAC (Índice de acceso y desarrollo cultural), propuesto por Melo y Peres (2004) y aplicado luego en procesos de investigación internacional (2007, 2009 y 2013). En tal sentido se crea el Índice desarrollo y acceso deportivo (IDAD).

El IDAD es un indicador, que basado en el principio de equidad, actúa como una forma de cálculo de la distribución de los escenarios deportivos, teniendo en cuenta variaciones demográficas. Es decir, para este caso, la distribución de la población en las comunas (forma de organización político-administrativa de la ciudad). El objetivo del IDAD es identificar si las oportunidades de acceso de las diferentes comunas y corregimientos de la ciudad a los escenarios deportivos son las mismas. Es por tanto un esfuerzo por mostrar posibles desigualdades a las que algunas poblaciones están expuestas en lo referente a oportunidades de acceso, considerando para ello, el lugar de residencia.

En resumen, la pregunta clave del IDAD es iserá que los habitantes de la comuna $X$ tienen la misma posibilidad de acceso a los escenarios deportivos que los habitantes de la comuna Y? Matemáticamente el IDAD consiste en dividir el número de escenarios, por el número de habitantes de una comuna o corregimiento. Es decir, el IDAD establece la cantidad de equipamientos por habitante.

Para calcularlo, el primer paso consiste en el diseño de una tabla en la que se relacionen las diversas comunas y corregimientos de la ciudad con los equipamientos existentes y el número de habitantes. El segundo paso consiste en calcular el IDAD dividiendo el número de escenarios en cada comuna por el número de habitantes.

Es decir:

$$
\begin{gathered}
\operatorname{IDAD} \text { Absoluto }(x)=\frac{\text { Total de escenarios }(x)}{\text { Total de habitantes }(x)} \\
0 \leq \operatorname{IDAD} \text { absoluto } \leq 1
\end{gathered}
$$

Donde $\mathrm{x}$ es la comuna.

El IDAD varía de 0 a 1 , donde 0 significa que no existe ningún escenario para la comunidad y uno, en una situación utópica, que existe un escenario para cada habitante. Lo que quiere decir que a mayor número de equipamientos por habitante, mayor será el IDAD absoluto.

El tercer paso hace referencia al IDAD relativo, que toma en cuenta la variación en la existencia de equipamientos entre las diversas comunas, teniendo como referencia, la que está en mejor condición.

EI IDAD relativo, se obtiene de dividir el IDAD absoluto de una determinada comuna, por el IDAD absoluto de la región de referencia (la que tiene el mejor IDAD absoluto) y multiplicarlo por 100). Es decir:

$\operatorname{IDAD}$ relativo $(\mathrm{x})=\frac{\operatorname{IDAD} \text { absoluto }(\mathrm{x})}{\operatorname{IDAD} \text { absoluto }(\mathrm{y})} \cdot 100$

Donde:

$x$ es la comuna

y es la comuna de referencia (la que tiene un mayor IDAD). 
En referencia al IDAD-absoluto, en la panorámica general de la distribución de los escenarios deportivos administrados por el INDER Medellín, como puede observarse en la Tabla 1, se presentan grandes diferencias entre algunas comunas. La situación que más llama la atención es la gran diferencia entre la comuna 5 y la 14. La primera, como la comuna con mayor cantidad de escenarios deportivos con 74 que equivale al $12,7 \%$ del total y el séptimo en IDH, y la Comuna 14 que siendo la primera en IDH, cuenta con la menor cantidad de escenarios, cinco, que representan el $0,86 \%$ de los escenarios administrados por el INDER Medellín en la zona urbana. De acuerdo con la proyección de población del municipio de Medellín para el 2015 el número de habitantes para este año es de 2.464.322. Es decir, que bajo la propuesta actual del INDER Medellín, existen 582 escenarios deportivos para casi 2.500 .000 habitantes.

\section{LOS ESCENARIOS DEPORTIVOS EN LA CIUDAD DE MEDELLÍN.}

El tema del tipo de escenarios, es importante pues da cuenta de las posibilidades que se ofrecen a la población. En este sentido podrán evidenciarse, a través de su identificación, las posibilidades de acceso en general o la restricción de la misma. Por ejemplo, la categoría escenario contiene en sí misma una mayor o menor posibilidad de acceso. No es lo mismo un escenario de ciudad, tipo estadio de fútbol, que una placa polideportiva, su vocación (competencia o tiempo libre), condiciona la posibilidad de acceso al mismo.

De acuerdo con el inventario hecho para la investigación, se encontraron 56 tipos diferentes de escenarios. El Gráfico 1 permitirá una mirada panorámica.

Gráfico 1- Distribución por comunas de los escenarios deportivos en la ciudad de Medellín

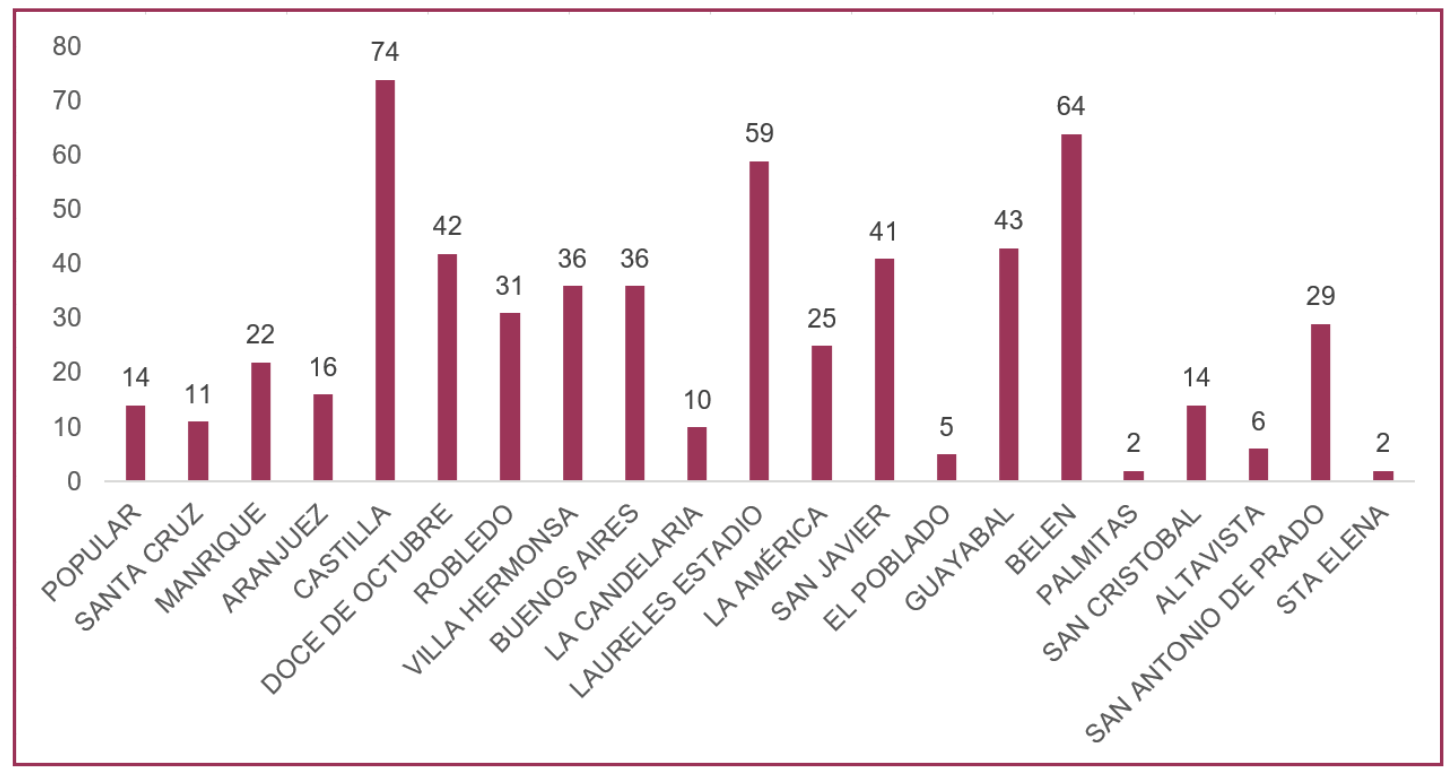

Fuente: construcción propia de los investigadores

La placa polideportiva, es el escenario que más aparece. Se registra 248 veces y aparece único con presencia en todas las comunas de la ciudad. Representa el 42,6\% de todos los escenarios que administra el INDER Medellín. Las canchas de fútbol, con 71, es decir el $12,2 \%$ de registros, aparecen como el segundo escenario en importancia de la ciudad, también con presencia en todas las comunas, con la excepción de Palmitas, que no registra este tipo de 
escenario. La Cancha/placa de microfútbol aparece con 36 registros, que equivalen al 6,2\% y las Placas/canchas de baloncesto que con 31 registros, representan el 5,3\% de los escenarios de la ciudad.

Este grupo de escenarios suman en conjunto 386 de los 582 bajo la administración del INDER Medellín, es decir, el 66,3\%, las 2/3 del total. En principio, esto hace visible una situación a tener en cuenta, que deberían recibir especial atención, tanto por su presencia en la ciudad, como por la característica de escenarios barriales. Representan una oportunidad para la gestión de procesos que permitan la generación de condiciones y oportunidades para el acceso de la población a los escenarios y a los beneficios que del uso se pueden obtener.

Un segundo tipo de escenarios, son las canchas sintéticas y las piscinas que aparecen con 27 registros cada una, es decir, el 4,6\% para cada caso. Este tipo de escenarios se han convertido por ejemplo para el primer caso, en lo que se denomina los desarrollos deportivos, que corresponden a escenarios con alguna infraestructura complementaria. Estos se registran en diez de las comunas de Medellín y entre las comunas 5, 15 y 16, con 15 registros, concentran el $55,5 \%$ de este tipo de escenarios.

En el caso de las piscinas, nueve comunas registran este tipo de escenario. Entre las comunas 3 con tres escenarios, la 5 con cuatro y la 11 con diez, concentran el $66,6 \%$ del total, es decir las $2 / 3$. La sola Comuna 11, posee diez piscinas, que equivalen al $37 \%$ de este escenario en la ciudad de Medellín.

El equipamiento Cancha de tenis se registra en 23 ocasiones, que equivalen al 3,91\% aproximadamente. Este escenario solo aparece en cuatro de las 21 comunas de la ciudad. Las comunas 5,11 y 16 , concentran el $95,6 \%$ de este tipo de escenarios.

Otros escenarios como las canchas de $\mathrm{Tejo}^{3}$, con 12 registros y las pistas de trote con 11 , representan otro tipo de equipamiento. En el caso del primero, se registran en ocho de las comunas. Y en el caso de las pistas de trote, en siete de las comunas de la ciudad, pero más de la mitad, $55,5 \%$, están concentradas en las Comunas 5 y 6 .

Luego aparecen algunos escenarios como Skate y Zona de Barras que se registran siete veces cada uno, es decir, el 1,2\% para cada caso y se distribuyen en cinco de las 21 Comunas. Para el primer caso, las Comunas de mayor presencia son la 5 y la 80 con dos de estos escenarios, mientras que las otras tres se registran en las Comunas, 1, 3 y 14.

El resto de los escenarios se concentran en el rango de uno a seis: cancha de minitenis y parques infantiles, con cinco, canchas de Voleybol y Gimnasios Urbanos, con cuatro Squash y con tres Muros de escalar, Cancha de Voleyplaya, ciclorutas, pistas de calentamiento y gimnasios.

A continuación, se presenta la distribución de los escenarios deportivos de acuerdo con las comunas que componen la ciudad (Gráfico 1). De acuerdo con el gráfico, las comunas con mayor cantidad de escenarios son en su respectivo orden Castilla con 74 que representan el 12,7\% del total; Belén con 64 o sea 11\% y Laureles-estadio con 59 es decir 10,1\%. Un segundo grupo lo componen las Comunas Guayabal con 43 escenarios, Doce de Octubre con 42 y San Javier con 41 , lo que equivale al 7,4\%, 7,21\% y 7\% respectivamente. Otro grupo

3 Es un juego que consiste en lanzar un disco metálico de aproximadamente 680 gramos a través de una cancha de arcilla de 18 metros de largo para hacer estallar las mechas (pequeños sobres con pólvora) que se encuentran en el bocín (círculo metálico que está ubicado en la cancha) y así ir sumando puntos. (Wikipedia. Disponible en:< https://es.wikipedia.org/wiki/Tejo (deporte)>. Acceso en: 25 mayo 2017. 
más, lo componen las Comunas Villa Hermosa y Buenos Aires con 36 escenarios cada una, que equivalen al 6,2\% para cada caso. Robledo con 31 representa el 5,32\% y San Antonio de Prado con 29 el 4,98\% del total. Con 22 escenarios que equivalen al 3,78\% está Manrique. Otro grupo, lo conforman las comunas que oscilan entre los 10 y 16 escenarios. Ellas son: Aranjuez con 16 escenarios, es decir, el 2,74\%; San Cristóbal y Popular poseen cada una 14 escenarios, que representan en los dos casos el $2.4 \%$ del total. Once escenarios registra Santa Cruz, que corresponde al 1,89\% de los escenarios. 10 escenarios registra La Candelaria, que equivale al $1,71 \%$ del total. Un último grupo por debajo de 10 escenarios, lo componen en su respectivo orden, Altavista que con seis que representa el 1,03\% del total. Le sigue El poblado con cinco que equivale al $0.86 \%$ de los escenarios administrados por el INDER Medellín. Por último están Palmitas y Santa Elena, con dos escenarios cada una, que representa en cada caso el 0,34\% del total.

\section{EL ÍNDICE DE ACCESO Y DESARROLLO DEPORTIVO IDAD ABSOLUTO}

De acuerdo con la Tabla 1, en la que se registran el IDAD Absoluto y el IDAD Relativo, se puede observar el registro de los escenarios deportivos en las comunas y corregimientos de la ciudad de Medellín, en relación con el número de habitantes y el comparativo entre ellas.

Tabla 1 - IDAD Absoluto, IDAD Relativo y su relación con indicadores de calidad de Vida.

\begin{tabular}{llcccccc}
\hline \multirow{2}{*}{ № } & \multicolumn{1}{c}{ Comuna } & Total & $\begin{array}{c}\text { NNa de } \\
\text { Habitantes }\end{array}$ & $\begin{array}{c}\text { IDAD } \\
\text { Absoluto }\end{array}$ & $\begin{array}{c}\text { IDAD } \\
\text { Relativo }\end{array}$ & $\begin{array}{c}\text { IDH }^{5} \\
\mathbf{2 0 1 1}\end{array}$ & IMCV $^{6}$ \\
\hline 1 & CASTILLA & 74 & 149,751 & 0,0005 & 100 & $86,26(7)$ & $47,5(8)$ \\
2 & LAURELES ESTADIO & 59 & 122,243 & 0,0005 & 97,56 & $93,75(2)$ & $70,1(2)$ \\
3 & GUAYABAL & 43 & 94,470 & 0,0004 & 92,1 & $86,79(6)$ & $52,0(6)$ \\
4 & BELEN & 64 & $196, .694$ & 0,0003 & 65,8 & $90,05(4)$ & $67,5(4)$ \\
5 & PALMITAS & 2 & 6,324 & 0,0003 & 63,9 & $78,32(21)$ & \\
6 & SAN JAVIER & 41 & 138,063 & 0,0003 & 60 & $81,88(15)$ & $39,2(12)$ \\
7 & SAN ANTONIO DE PRADO & 29 & 108,856 & 0,0002 & 53,8 & $83,49(10)$ & \\
8 & BUENOS AIRES & 36 & $136, .774$ & 0,0002 & 53,2 & $85,41(8)$ & $49,1(7)$ \\
9 & VILLA HERMOSA & 36 & 137,531 & 0,0002 & 52,84 & $82,28(14)$ & $38,0(14)$ \\
10 & LA AMÉRICA & 25 & 96,278 & 0,0002 & 52,4 & $91,80(3)$ & $63,0(3)$ \\
11 & DOCE DE OCTUBRE & 42 & 193,657 & 0,0002 & 43,7 & $83,47(12)$ & $40,3(11)$ \\
12 & ROBLEDO & 31 & 171,660 & 0,0002 & 36,4 & $83,80(10)$ & $45,1(9)$ \\
13 & SAN CRISTOBAL & 14 & 79,458 & 0,0002 & 35,6 & $82,52(13)$ & \\
14 & ALTAVISTA & 6 & $36, .463$ & 0,0002 & 35,2 & $81,31(16)$ & \\
15 & MANRIQUE & 22 & 159,658 & 0,0001 & 27,7 & $81,20(17)$ & $38,4(13)$ \\
16 & LA CANDELARIA & 10 & 85,505 & 0,0001 & 23,50 & $87,99(5)$ & $57,0(5)$ \\
17 & STA ELENA & 2 & 18,025 & 0,0001 & 22,2 & $80,86(18)$ & \\
18 & POPULAR & 14 & 130,362 & 0,0001 & 21,6 & $78,73(20)$ & $32,8(16)$ \\
19 & ARANJUEZ & 16 & $162, . .252$ & 0,00009 & 19,9 & $83,91(9)$ & $42,5(10)$ \\
20 & SANTA CRUZ & 11 & 111,452 & 0,00009 & 19,4 & $80,60(19)$ & $35,9(15)$ \\
21 & EL POBLADO & 5 & 128,839 & 0,00004 & 7,8 & $97,32(1)$ & $75,9(1)$ \\
\hline & & Fuente: construcción propia de los investigadores & & & \\
& & 7 & & & &
\end{tabular}

Para el caso del IDAD Absoluto, Castilla aparece en el primer lugar, con 0,00049 ( $7^{\circ}$ IDH). Le sigue Laureles-Estadio con 0,00048 (2 IDH), Guayabal con 0,00045 (6익), Belén 
con $0,000325\left(4^{\circ}\right.$ IDH) y Palmitas con $0,000316\left(21^{\circ}\right.$ IDH). Un segundo grupo lo compone San Javier con 0,000296 (15익), San Antonio de Prado con 0,000266 (10ㅇIDH), Buenos Aires con 0,000263 ( $8^{\circ}$ IDH), Villa Hermosa con 0,000261 (14ㅇIDH), La América con 0,000259 (3 IDH) y Doce de Octubre con un IDAD absoluto de 0,000216 (12 IDH). Un tercer grupo está compuesto por Robledo con 0,000180 (10ㅇIDH), San Cristóbal con 0,000176 y Altavista con 0,000174 (para ellos no aparece IDH. Corresponden a la zona rural). Le sigue Manrique

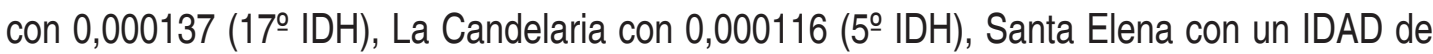
0,000110 (Sin IDH. Zona rural) y Popular con 0,000107 (20 IDH). Un último grupo lo componen Aranjuez con un IDAD de 0,0000986 (9ㅇIDH), Santa Cruz cuyo IDAD es 0,0000960 (19ㅇIDH) y El Poblado con un IDAD de 0,0000388 (1익).

En relación con lo anterior, emergen dos asuntos que vale la pena plantear, para hacer visibles algunas situaciones particulares. El primer caso, tiene que ver con algunos corregimientos con poca población, lo que lleva a que cuando se relaciona con los pocos escenarios deportivos que tienen, registran un IDAD más alto que en otras Comunas con mayor número de escenarios, pero de igual manera con una mayor población. El segundo caso, hace referencia a la coincidencia entre el IDAD y el IDH, como en el caso de las Comunas 11, 15 y 16 , donde los el IDAD y el IDH están equilibrados. Otra situación es la que se presenta con comunas que aparecen en los primeros puestos del IDAD como en el caso de Castilla, con el mayor IDAD, y en puestos intermedios de IDH y la Comuna 13 San Javier, que ocupa el 6ำ lugar en el IDAD y el 15 en IDH. Un grupo más, es el que conforman las Comunas Popular y Santa Cruz que se registran entre las últimas tanto en el IDAD $18^{\circ}$ y $20^{\circ} \mathrm{como}$ en el IDH $\left(20^{\circ}\right.$ y 19을 respectivamente). Un caso especial, es el que registra El Poblado, pues siendo la primera Comuna en calidad de vida, aparece con el IDAD más bajo en la zona urbana, superada solo por los Corregimientos de Palmitas y Santa Elena.

De esta manera, la relación entre escenarios deportivos y calidad de vida no es tan directa como pudiera parecer. En esta dirección, además de índices como el IDAD, será necesario avanzar en la construcción de indicadores e índices que relacionen los diferentes factores que condicionan el buen vivir de los habitantes de la ciudad. Es decir, ir más allá de los tradicionales indicadores, que desde una perspectiva instrumental, consideran que el número de escenarios deportivos es evidencia suficiente para el bienestar.

\section{5 ÍNDICE DE ACCESO Y DESARROLLO DEPORTIVO RELATIVO. IDAD RELATIVO}

De acuerdo con este indicador, el mejor IDAD, lo tiene Castilla con 0.00049 , que para nuestro caso como Comuna referente, será el 100\%. En comparación con las otras Comunas y Corregimientos, el porcentaje que requieren para tener el mismo acceso a escenarios deportivos es el siguiente: Laureles Estadio requeriría poco menos de 2,5\% para igualar a la Comunas referente y Guayabal poco menos del 8\%. Las Comunas Belén y San Javier y el Corregimiento de Palmitas necesitan en el primer caso del $40 \%$, y en los últimos de un poco menos del $46 \%$. Para el caso de la Comuna Doce de Octubre, equivale a poco menos de $58 \%$.

Otro grupo lo componen Las comunas Robledo y los Corregimientos de San Cristóbal y Altavista; la primera con un déficit del casi el $63 \%$ y los segundos del $66 \%$. La Comuna Manrique presenta una desventaja de casi del $72 \%$ y La Candelaria, Santa Elena y Popular entre el $76 \%$ y el $79 \%$. Por último, las Comunas de Aranjuez y Santa Cruz casi del $80 \%$ y 
El Poblado con un déficit cercano al 94\%, con relación a Castilla. Este último dato es muy significativo, pues la Comuna 14, la primera en el IDH (Índice de Desarrollo Humano y el IMCD 4 (Índice Multidimensional de Condiciones de Vida) aparece de última en el IDAD. Un hallazgo que abre un importante camino, en la posible relación entre escenarios deportivos y calidad de vida. Aunque es necesario aclarar que la presente investigación buscó dar cuenta de los escenarios públicos administrados por el INDER Medellín y no se tuvieron en cuenta los escenarios privados.

\section{CONSIDERACIONES FINALES}

De acuerdo con lo visto en este componente del desarrollo de la investigación, el acceso representa uno de los aspectos con mayor relevancia en términos de posibilidad para beneficiarse de los escenarios deportivos.

Si bien indicadores como los metros cuadrados en relación con el número de habitantes pueden dar una idea de la situación, elementos como la calidad de vida, los niveles de pobreza y violencia, entre otros, no son visibles. EI IDAD permite, a partir de cada comuna, establecer la posibilidad real de acceso, mediante la comparación entre el tipo escenario deportivo, su cantidad y el número de habitantes. En esta dirección, ejercicios como este pueden servir como línea base, para procesos de gestión de la 5administración municipal. Así, la propuesta deja ver otros asuntos como las condiciones de vida y el contexto social, para una lectura más pertinente de la real incidencia de los escenarios deportivos en la vida de las personas. Es decir, la sola presencia de un escenario no es condición suficiente para una lectura del bienestar humano.

De acuerdo con lo anterior, más que indicadores que responden a una definición dada predeterminada, que condiciona de entrada su lectura, debe apostarse por aquellos que identifiquen las condiciones de posibilidad de los contextos. Al respecto, indicadores como el IDAD, ofrecen la posibilidad de comparación con otras temáticas relacionadas con la calidad de vida.

En el caso de los escenarios, uno de los aspectos que mostró esta parte de lainvestigación, es que el equipamiento, en sí mismo, no es un recorte suficiente de la realidad en temas de bienestar social, pues desconoce la realidad concreta de las comunas y corregimientos. Sobre esto, es muy ilustrativo el hecho de que la Comuna 5 que ocupa el primer lugar en el IDAD, se ubiqué en el séptimo del IDH. Mientras que la Comuna 16 El Poblado, aparezca en el primer lugar del IDH y en el último del IDAD. Esto muestra lo problemático de relacionar directamente los escenarios deportivos con temas como paz, convivencia, inclusión, bienestar, etc.

Otro aspecto que hizo visible este componente de la investigación, fue que las dos terceras partes de los escenarios, es decir el $66 \%$, corresponden a escenarios barriales tales como placas polideportivas, placas de baloncesto, microfútbol y voleibol, que representan oportunidades para la mayoría de las personas en los barrios. Por tanto deberían tener una mayor atención e inversión por parte de la administración municipal, por corresponder a escenarios destinados al tiempo libre de los habitantes y no a procesos de competencia. En últimas, el IDAD representa un importante apoyo para construcción de la política pública del deporte y la recreación en el ámbito municipal. 
En todo caso, aportó a la posibilidad de una lectura de la relación entre los escenarios deportivos y sus posibilidades de contribución al bienestar. En este sentido, la relación directa entre estos y las condiciones de vida, debe ser problematizada a partir de procesos de diversa índole que lo ubiquen en su justa medida. Temas de barreras, juegos de poder de diferentes actores, necesidad de favorecer formas democráticas de acceso e iniciativas que busquen su cualificación, mediante la ampliación de condiciones y posibilidades para el disfrute efectivo de esos territorios.

\section{REFERENCIAS}

GUBA, Egon G.; LINCOLN, Yvonna L. Paradigmas en competencia en la investigación cualitativa. In: DENMAN, Catalina; HARO, Jesús Armando. Por los rincones. Antología de métodos cualitativos en la investigación social. Sonora: Colegio de Sonora, 2002. p. 113-145.

INFORME de calidad de vida de Medellín 2012-2015. Medellin, 2015. (Serie Cómo vamos, 10 años).

INSTITUTO DE DEPORTES Y RECREACIÓN DE MEDELLÍN (INDER). Escenarios deportivos y recreativos INDER Medellín: espacios públicos que transforman ciudad. Disponible en: < https:// issuu.com/hugoalzate/docs/escenarios deportivos y recreativos >. Acceso en: 20 ago. 2015.

INSTITUTO DE DEPORTES Y RECREACIÓN DE MEDELLÍN (INDER). Usos y prácticas de los escenarios deportivos administrados por el INDER Medellín año 2012. Medellín, 2013.

INSTITUTO DE DEPORTES Y RECREACIÓN. Sistema de indicadores sociales INDER Alcaldía de Medellín, 2009-2010. Disponible en: <https://pt.scribd.com/document/278804128/ Presentacion-Indicadores-en-Torno-a-Actividad-Fisica-Deporte-y-Recreacion-2010>. Acceso en: 25 mayo 2017.

MELO, Victor Andrade de; PERES, Fabio de Faria (Coord.). Equipamentos culturais na América do Sul: desigualdades. Rio de Janeiro: Apicure, 2009.

MELO, Victor Andrade de; PERES, Fabio de Faria. A cidade e o lazer: as desigualdades sócioespaciais na distribuição dos equipamentos culturais na cidade do Rio de Janeiro e a construção de um indicador que oriente as ações em políticas públicas. Movimento, v. 11, n. 3, p. 127-151, set./dez. 2005.

MOLINA BEDOYA, Victor Alonso et al. Ocio y ciudad: diálogos para la construcción de espacios lúdicos Colombia. Medellín: CIVITAS, 2007.

MUSSINO, Antonio. Statistica e Sport: non solo numeri. Roma: Società Stampa Sportiva. 1997.

OLIVEIRA, Ailton Fernando Santana de; TAFFAREL, Celi Nelza; BELEM, Cristiano. Infraestrutura esportiva: desenvolvimento de metodologias. Revista Brasileira de Ciências do Esporte, v. 36, n. 2, supl., p. S617- S635, abr. /jun. 2014.

ROTH DEUBEL, André-Noel. Perspectivas teóricas para el análisis de las políticas públicas: ¿de la razón científica al arte retórico? Estudios Políticos, n. 33, p. 67-91, jul./dic. 2008.

STUART RENDÓN, Patricia Elena. La práctica deportiva y su relación directa con el uso de los escenarios públicos y privados del barrio Castilla de la ciudad de Medellín. 2011. 
114f. Trabajo de Grado. Disponible en: <http://viref.udea.edu.co/contenido/pdf/270-lapractica.pdf>. Acceso en: 25 sep. 2015.

TABARES FERNÁNDEZ, José Fernando; MOLINA BEDOYA , Victor (Org.). Desigualdades sociespaciales en la distribución de los equipamientos culturales en América latina. Medellín: Funámbulos, 2013.

ZEMELMAN MERINO, Hugo. Crítica epistemológica de los indicadores. México: El Colegio de México, 1989.

\section{Apoyo:}

La investigación fue financiada por el Instituto de Deportes y Recreación de Medellín, mediante contrato C-01936-15 con la Universidad de Antioquia.

\section{Agradecimientos:}

En la investigación participaron Alejandro Escobar Chavarriaga, Elkin de Jesús Vergara Marín, Carlos Esneider Patiño Torres por el Grupo de Investigación Ocio, Expresiones Motrices y Sociedad de la Universidad de Antioquia y Carlos Arturo Gómez Valencia por el Instituto de Deporte y Recreación de Medellín (INDER). Como estudiantes estuvieron Jhon Esteban Rivera 